large inherent dependence on temperature, we concluded that the folding reaction is at least near the Kramers high friction limit.

\section{Computational}

The double-well potential used was $g(x)=x^{4}-2 x^{2}$. Temperature effects were treated as a linear bias along the reaction coordinate, $t(x, T)=A(T) x$, where $A(T)$ is an adjustable parameter for matching the equilibrium data. The folded and unfolded states are separated by 2 distance units along the reaction coordinate, corresponding to a typical helix diffusion length when taken to be nanometres. The population at $x<0.83$ was assumed to have the same fluorescence signature as the unfolded state, and at $x>0.83$ as the folded state (for compatibility with the three-well model in the Supplementary Information, any value $x>0$ yields the same qualitative result). Fluorescence was simulated by convolving populations (for example Fig. $4 \mathrm{~b}$ ) with this response. The onedimensional Langevin equation with gaussian white noise was integrated by using a fourth-order Runge-Kutta method. A time-step size of 0.01 was used in the integration, and time steps were scaled to match the experimentally observed absolute kinetics. Similar calculations for a three-well model that also matches the data are described in the Supplementary Information.

\section{Received 24 January; accepted 31 March 2003; doi:10.1038/nature01609.}

1. Berne, B. J. in Activated Barrier Crossing: Applications in Physics, Chemistry and Biology (eds Hänggi, P. \& Fleming, G. R.) 82-119 (World Scientific, Singapore, 1993).

2. Hagen, S. J., Hofrichter, J., Szabo, A. \& Eaton, W. A. Diffusion-limited contact formation in unfolde cytochrome c: Estimating the maximum rate of protein folding. Proc. Natl Acad. Sci. USA 93, 11615-11617 (1996).

. Lapidus, L. J., Eaton, W. A. \& Hofrichter, J. Measuring the rate of intramolecular contact formation in polypeptides. Proc. Natl Acad. Sci. USA 97, 7220-7225 (2000).

4. Bieri, O. et al. The speed limit for protein folding measured by triplet-triplet energy transfer. Proc. Natl Acad. Sci. USA 96, 9597-9601 (1999).

5. Yeh, I. C. \& Hummer, G. Peptide loop-closure kinetics from microsecond molecular dynamics simulations in explicit solvent. J. Am. Chem. Soc. 124, 6563-6568 (2002).

6. Thirumalai, D. Time scales for the formation of the most probable tertiary contacts in proteins with applications to cytochrome C. J. Phys. Chem. B 103, 608-610 (1999).

7. Portman, J. J., Takada, S. \& Wolynes, P. G. Microscopic theory of protein folding rates. II. Local reaction coordinates and chain dynamics. J. Chem. Phys. 114, 5082-5096 (2001).

8. Portman, J. J., Takada, S. \& Wolynes, P. G. Microscopic theory of protein folding rates. I. Fine structure of the free energy profile and folding routes from a variational approach. J. Chem. Phys. 114, 5069-5081 (2001).

9. Hagen, S. J., Hofrichter, J. \& Eaton, W. A. Rate of intrachain diffusion of unfolded cytochrome c. J. Phys. Chem. B 101, 2352-2365 (1997)

10. Schuler, B., Lipman, E. A. \& Eaton, W. A. Probing the free-energy surface for protein folding with single-molecule fluorescence spectroscopy. Nature 419, 743-747 (2002).

11. Gruebele, M., Sabelko, J., Ballew, R. \& Ervin, J. Laser temperature jump induced protein refolding. Acc. Chem. Res. 31, 699-707 (1998).

12. Metzler, R., Klafter, J. \& Jortner, J. Hierarchies and logarithmic oscillations in the temporal relaxation patterns of proteins and other complex systems. Proc. Natl Acad. Sci. USA 96, 11085-11089 (1999).

13. Burton, R. E., Huang, G. S., Daugherty, M. A., Calderone, T. L. \& Oas, T. G. The energy landscape of a fast-folding protein mapped by Ala $\rightarrow$ Gly substitutions. Nature Struct. Biol. 4, 305-309 (1997).

14. Ghaemmaghami, S., Word, J. M., Burton, R. E., Richardson, J. S. \& Oas, T. G. Folding kinetics of a fluorescent variant of monomeric lambda represssor. Biochemistry 37, 9179-9185 (1998).

15. Myers, J. M. \& Oas, T. G. Contribution of a buried hydrogen bond to lambda repressor folding kinetics. Biochemistry 38, 6761-6768 (1999).

16. Chang, I. J., Lee, J. C., Winkler, J. R. \& Gray, H. B. The protein-folding speed limit: intrachein diffusion times set by electron transfer rates in denatured $\mathrm{Ru}\left(\mathrm{NH}_{3}\right)_{5}($ His-33)-Zn-cytochrome c. Proc. Natl Acad. Sci. USA 100, 3838-3840 (2003).

17. Silow, M. \& Oliveberg, M. Transient aggregates in protein folding are easily mistaken for folding intermediates. Proc. Natl Acad. Sci. USA 94, 6084-6086 (1997).

18. Sabelko, J., Ervin, J. \& Gruebele, M. Observation of strange kinetics in protein folding. Proc. Natl Acad Sci. USA 96, 6031-6036 (1999).

19. Garcia-Mira, M. M., Sadqi, M., Fischer, N., Sanchez-Ruiz, J. M. \& Muñoz, V. Experimental identification of downhill protein folding. Science 298, 2191-2195 (2002).

20. Silow, M. \& Oliveberg, M. High-energy channeling in protein folding. Biochemistry 36, 7633-7637 (1997).

21. Pappenberger, G., Saudan, C., Becker, M., Merbach, A. E. \& Kiefhaber, T. Denaturant-induced movement of the transition state of protein folding revealed by high-pressure stopped-flow measurements. Proc. Natl Acad. Sci. USA 97, 17-22 (2002).

22. Snow, C. D., Nguyen, H., Pande, V. S. \& Gruebele, M. Absolute comparison of simulated and experimental protein-folding dynamics. Nature 420, 102-106 (2002).

23. Mayor, U. et al. The complete folding pathway of a protein from nanoseconds to microseconds. Nature 421, 863-867 (2003).

24. Ervin, J., Sabelko, J. \& Gruebele, M. Submicrosecond real-time fluorescence detection: application to protein folding. J. Photochem. Photobiol. B 54, 1-15 (2000).

Supplementary Information accompanies the paper on www.nature.com/nature.

Acknowledgements We thank T. Oas for suggesting many helpful lambda repressor mutations This work was supported by an Alumni Scholarship and a Camille Dreyfus Teacher-Scholar Award to M.G.

Competing interests statement The authors declare that they have no competing financial interests.

Correspondence and requests for materials should be addressed to M.G. (gruebele@scs.uiuc.edu).

erratum

\title{
Antibody neutralization and escape by HIV-1
}

Xiping Wei, Julie M. Decker, Shuyi Wang, Huxiong Hui, John C. Kappes, Xiaoyun Wu, Jesus F. Salazar-Gonzalez, Maria G. Salazar, J. Michael Kilby, Michael S. Saag, Natalia L. Komarova, Martin A. Nowak, Beatrice H. Hahn, Peter D. Kwong \& George M. Shaw

Nature 422, 307-312 (2003).

In the seventh panel of Fig. 2 of this Letter, the V5 sequence of clone 391-3 appeared incorrectly as: SEKDQTEIFRP. It should read: SKDNQTEIFRP. In addition, there should be no yellow shading (indicating a change in glycosylation) for this sequence.

\section{corrigenda}

\section{Synaptic depression in the localization of sound}

Daniel L. Cook, Peter C. Schwindt, Lucinda A. Grande \& William J. Spain

Nature 421, 66-70 (2003)

It has come to our attention that we failed to cite a relevant study ${ }^{1}$ in our Letter. These authors identified the mechanism of synaptic depression measured at the embryonic chick nucleus magnocellularis to nucleus laminaris synapse as primarily presynaptic, which justifies the synaptic depletion model we used. Furthermore, the narrowing of coincidence detection time windows with EPSP depression as they observed may contribute to the adaptive mechanisms that we described.

1. Kuba, H., Konomi, K. \& Ohmori, H. Eur. J. Neurosci. 15, 984-990 (2002).

\section{Short interfering RNA confers intracellular antiviral immunity in human cells}

\section{Leonid Gitlin, Sveta Karelsky \& Raul Andino}

Nature 418, 430-434 (2002).

In Fig. 5a of this Letter, the first and third panels (untreated and siL-treated cells, respectively) should not be identical: the correct figure is shown here.

a

$\mathrm{MOI}=10$ 24 h.p.i.
Untreated

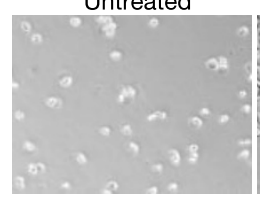

$\mathrm{siC}$

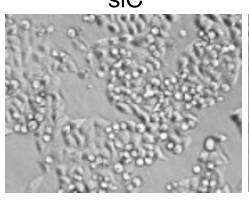

sil

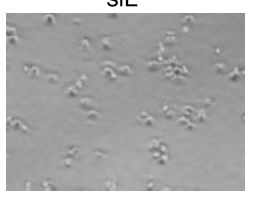

